\title{
One-year survival of patients with high-grade glioma discharged alive from the intensive care unit
}

\author{
Maxens Decavèle ${ }^{1,2}\left(\mathbb{D} \cdot\right.$ Nicolas Gatulle $^{1} \cdot$ Nicolas Weiss $^{3,4} \cdot$ Isabelle Rivals $^{2,5} \cdot$ Ahmed Idbaih $^{6} \cdot$ Sophie Demeret $^{3}$. \\ Julien Mayaux ${ }^{2} \cdot$ Martin Dres $^{1,2} \cdot$ Elise Morawiec $^{2} \cdot$ Khe Hoang-Xuan $^{6} \cdot$ Thomas Similowski $^{1,2} \cdot$ Alexandre Demoule $^{1,2}$
}

Received: 12 July 2020 / Revised: 24 August 2020 / Accepted: 26 August 2020 / Published online: 29 August 2020

(c) Springer-Verlag GmbH Germany, part of Springer Nature 2020

\begin{abstract}
Introduction Only limited data are available regarding the long-term prognosis of patients with high-grade glioma discharged alive from the intensive care unit. We sought to quantify 1-year mortality and evaluate the association between mortality and (1) functional status, and (2) management of anticancer therapy in patients with high-grade glioma discharged alive from the intensive care unit.

Patients and methods Retrospective observational cohort study of patients with high-grade glioma admitted to two intensive care units between January 2009 and June 2018. Functional status was assessed by the Karnofsky Performance Status. Anticancer therapy after discharge was classified as (1) continued (unchanged), (2) modified (changed or stopped), or (3) initiated (for newly diagnosed disease).

Results Ninety-one high-grade glioma patients (73\% of whom had glioblastoma) were included and 78 (86\%) of these patients were discharged alive from the intensive care unit. Anticancer therapy was continued, modified, and initiated in $41 \%, 42 \%$, and $17 \%$ of patients, respectively. Corticosteroid therapy at the time of ICU admission [odds ratio (OR) 0.07] and cancer progression (OR 0.09) was independently associated with continuation of anticancer therapy. The mortality rate 1 year after ICU admission was 73\%. On multivariate analysis, continuation of anticancer therapy (OR 0.18) and Karnofsky performance status on admission (OR 0.90) were independently associated with lower 1-year mortality.

Conclusion The presence of high-grade glioma is not sufficient to justify refusal of intensive care unit admission. Performance status and continuation of anticancer therapy are associated with higher survival after intensive care unit discharge. Previous presentation Preliminary results were presented at the most recent congress of the French Intensive Care Society, Paris, 2019.
\end{abstract}

Keywords Malignant brain tumors · Glioma · One-year survival · Intensive care unit · Anticancer therapy · Performance status

\section{Introduction}

Patients with solid tumor or hematologic malignancies account for $20 \%$ of intensive care unit (ICU) admissions [1, 2]. Because the prognosis of cancer patients is similar to that of non-cancer patients [1,3], a diagnosis of cancer should not preclude ICU admission. This general rule also applies

Electronic supplementary material The online version of this article (https://doi.org/10.1007/s00415-020-10191-0) contains supplementary material, which is available to authorized users.

Maxens Decavèle

maxens.decavele@aphp.fr

Extended author information available on the last page of the article to patients with primary malignant brain tumors admitted to the ICU [4].

The outcome of patients with primary malignant brain tumors has been described in terms of short-term and medium-term mortality [4-6]. However, data on 1-year mortality in these patients discharged alive from the ICU are lacking [3-7], and previous series included mixed highgrade gliomas (HGG), low-grade gliomas, and primary central nervous system lymphomas which have a heterogeneous prognosis [4-6]. In addition, the impact of an ICU stay on health-related performance status and the opportunity to continue anticancer therapy remains unclear [8-11]. These last two points are of utmost importance, as a marked reduction of performance status is commonly observed in patients 
requiring mechanical ventilation or vasopressors [12-16]. In turn, this poor performance status at ICU discharge may jeopardize long-term outcome by postponing or canceling anticancer therapy $[10,11]$. This risk is particularly high in patients with primary malignant brain tumors, as these tumors are known to reduce performance status [17-19], especially in patients with HGG.

We designed the present study to identify factors associated with 1-year outcomes in patients with HGG who survived an unplanned medical ICU stay. In addition, we examined changes in performance status and changes in the management of anticancer therapy after ICU discharge. This study focused on a homogeneous population of HGG, corresponding to majority of primary brain tumors with the most severe prognosis and raising the most challenging decisions concerning ICU admission. Our hypotheses were that, among HGG patients discharged alive from the ICU: (1) a substantial proportion of patients would still be alive 1 year after ICU discharge, with relatively good performance status, (2) anticancer therapy could be continued in a substantial proportion of patients, and (3) the performance status at ICU admission and maintenance of anticancer therapy were associated with a higher 1-year survival rate.

\section{Patients and methods}

\section{Study design and settings}

The study was conducted from January 2009 to June 2018 in two medical ICUs: a 16-bed ICU in a pulmonology department and a 16-bed ICU in a neurology department. Both ICUs are located in a university hospital with a strong neurological orientation including a specific neuro-oncology department (about 500 newly diagnosed patients each year) and the national reference center for high-grade oligodendroglial tumor (i.e., POLA Network). This study was approved by the French Intensive Care Society Institutional Review Board (CE SRLF 20-15) and information was given to the patients or their relatives. Data from this cohort have been previously published $[4,6]$.

\section{Patient selection}

Data were extracted from a prospectively managed database that comprehensively describes all patient stays in the two ICUs (Fusion, Varimed, France). The database of the two ICUs comprised 6,437 records, corresponding to $100 \%$ of admissions over the study period. In patients with several readmissions, only the first stay was included in the analysis. This set of 6,437 records was retrospectively searched for all consecutives cases of HGG, defined as grade III (anaplastic astrocytoma and oligodendroglioma) and grade IV (glioblastoma) glioma according to the 2016 World Health Organization (WHO) Classification of Tumors of the Central Nervous System [20]. Patient who underwent recent neurosurgery $(<2$ weeks) or any other recent surgery $(<4$ weeks) and patients under the age of 18 years were excluded.

\section{Data collection}

At the time of admission, gender, age, comorbidities using the Charlson Comorbidity Index (CCI) [21], physiological variables such as body temperature, respiratory rate, heart rate, systolic blood pressure, and Glasgow coma scale and various laboratory variables were recorded. Severity on admission was assessed by the Simplified Acute Physiology Score (SAPS) II [22] and the Sequential Organ Failure Assessment (SOFA) [23]. Performance status was assessed during the week before ICU admission and 1, 3, 6, 9, and 12 months after ICU admission, using the Karnofsky Performance Status Scale [24]. The tumor type was determined histologically on either the resection specimen or a biopsy. IDH $1 / 2$ mutation and $1 \mathrm{p} / 19 \mathrm{q}$ codeletion molecular status were also collected when available (systematic testing in our center since 2013). The reason for admission was determined retrospectively from the conclusions of the medical records. In case of admission for coma, the diagnosis of seizures was adopted when abnormal movements highly suggestive of seizures were observed, with or without electroencephalographic confirmation, or in the absence of suggestive movements, by consciousness alteration associated with electroencephalographic confirmation of seizures. Cancer disease status was classified as controlled (partial response, complete response, or stable disease), in progression, or newly diagnosed when the cancer was diagnosed during or after ICU admission or when the cancer was diagnosed during the 2 weeks preceding the ICU stay and no anticancer therapy had yet been delivered. Anticancer therapy after ICU discharge was classified as follows: (1) continued, when the anticancer therapy planned and initiated before ICU admission was continued unchanged after ICU discharge, (2) modified, when the anticancer therapy planned and initiated before ICU admission was changed or stopped after ICU discharge, and (3) initiated, when, for patients with newly diagnosed cancer, anticancer therapy was initiated during or after the ICU stay. Anticancer therapy only comprised chemotherapy and radiation therapy. We also recorded whether or not patients were receiving corticosteroid therapy at the time of ICU admission. The presence of corticosteroids at admission was not considered to constitute anticancer therapy. Finally, advanced life support measures taken during the ICU stay and vital status 1 year after ICU admission (1-year mortality) were recorded. 


\section{Statistical analysis}

Continuous variables were reported as median and interquartile interval, and categorical variables were reported as frequencies (\%). Categorical variables were compared using the Chi-square test or Fisher's exact test, as appropriate. Continuous variables were compared using the Mann-Whitney test or the Kruskal-Wallis test. All tests were two-sided and $p$ values $<0.05$ were considered statistically significant. Multivariate logistic regression was performed to identify factors associated with one-year mortality after ICU admission. In patients receiving anticancer therapy prior to admission, multivariate logistic regression was performed to identify factors associated with continuation of anticancer therapy. Factors yielding $p$ values $<0.20$ or considered to be clinically relevant were entered in the model and missing data (1.8\%) were imputed by the nearest-neighbor method. Odds ratios (ORs) and their 95\% confidence intervals (CI) were calculated for significant factors. One-year survival according to continuation of anticancer therapy after ICU discharge was evaluated using Kaplan-Meier survival function estimates. The impact of anticancer therapy on survival was assessed with the log-rank test.

The Karnofsky performance status was analyzed using a linear mixed model with anticancer therapy and times as fixed-effect factors, and the patient as random-effect factor. The linear mixed model was fitted with the restricted maximum-likelihood method. Post hoc tests of significance of the fixed-effect factor between pairs of conditions were performed with a likelihood ratio test.

Statistical analyses were performed using $\mathrm{R}$ version 3.5.2. and Matlab version 9.6.0.1150989 (R2019a).

\section{Results}

Figure 1 displays the study flowchart. Of the 91 patients included, 58 (64\%) were admitted to the medical ICU and $33(36 \%)$ were admitted to the neurological ICU.

\section{Patient characteristics}

The diagnosis of HGG was confirmed histologically in all patients and was based on examination of the surgical resection specimen for $33(36 \%)$ patients or a biopsy specimen for $58(64 \%)$ patients. The main characteristics of the 91 patients are displayed in Table 1. Tumor types were distributed as follows: 66 (73\%) glioblastomas (grade IV), 15 (16\%) anaplastic astrocytomas (grade III), and $10(11 \%)$ anaplastic oligodendrogliomas (grade III). The cancer diagnosis was initiated or established during the ICU stay for $15(16 \%)$ patients and was established prior to ICU admission for the remaining 76 (84\%) patients; median time between cancer diagnosis and ICU admission was 6 (2-20) months. ICU and

Fig. 1 Study flowchart

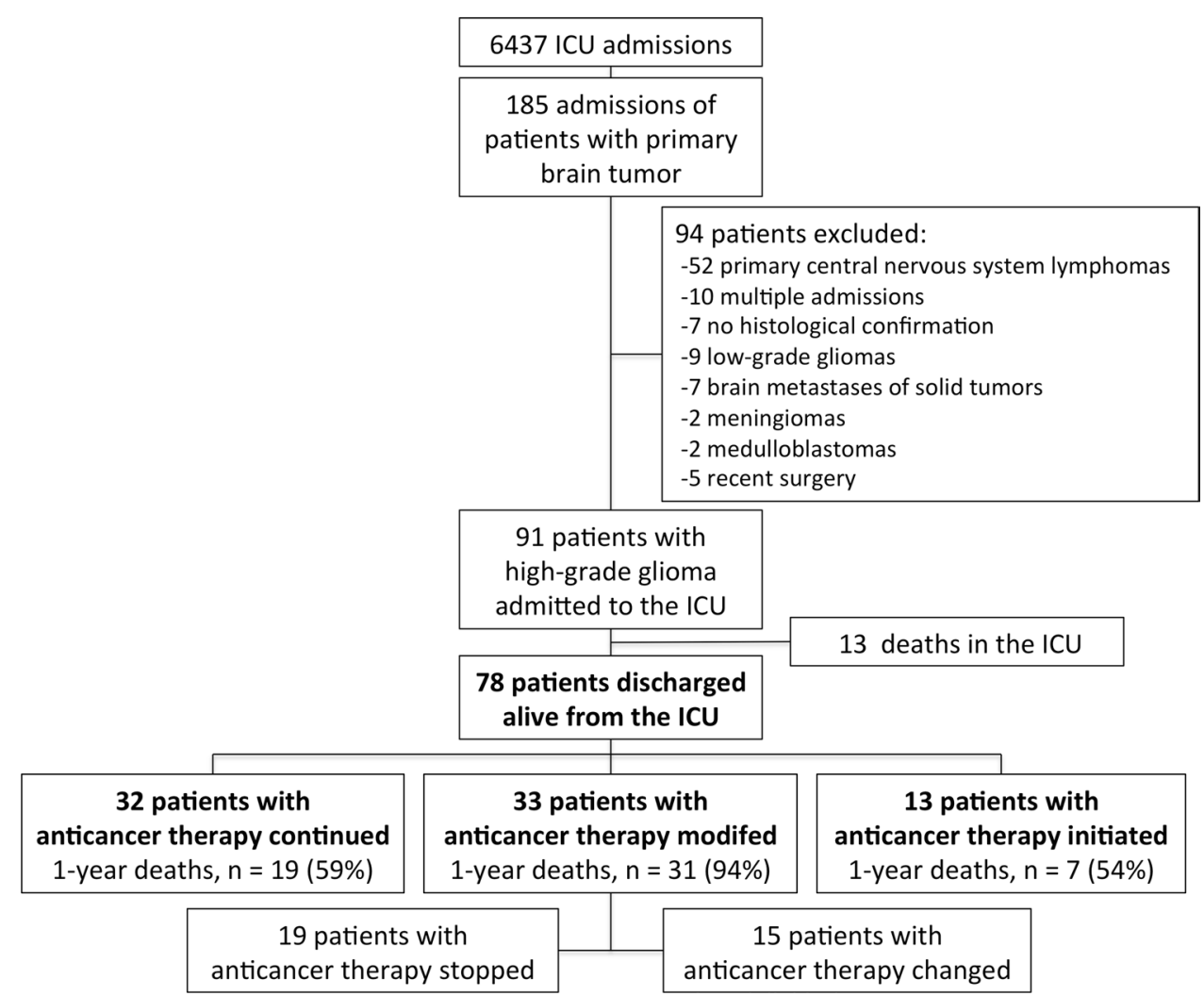


Table 1 Characteristics of the 91 patients at the time of intensive care unit (ICU) admission

\begin{tabular}{|c|c|}
\hline \multicolumn{2}{|l|}{ Variables } \\
\hline Age, years & $57(44-67)$ \\
\hline Gender (male), $n(\%)$ & $56(62)$ \\
\hline \multicolumn{2}{|l|}{ Comorbidities } \\
\hline Chronic heart failure, $n(\%)$ & $4(4)$ \\
\hline Chronic respiratory disease, $n(\%)$ & $7(8)$ \\
\hline Charlson comorbidity index & $3(2-4)$ \\
\hline Karnofsky performance status at admission, (\%) & $70(60-85)$ \\
\hline \multicolumn{2}{|l|}{ Disease status at admission } \\
\hline Cancer progression, $n(\%)$ & $48(53)$ \\
\hline Newly diagnosed, $n(\%)$ & $15(16)$ \\
\hline Controlled, $n(\%)$ & $28(31)$ \\
\hline Grade IV, $n(\%)$ & $66(73)$ \\
\hline IDH1/2 mutation, $n(\%)$ & $8(17)$ \\
\hline $1 \mathrm{p} / 19 \mathrm{q}$ codeletion, $n(\%)$ & $2(7)$ \\
\hline \multicolumn{2}{|l|}{ Anticancer therapy received at admission } \\
\hline Chemotherapy, $n(\%)$ & $64(70)$ \\
\hline Radiotherapy, $n(\%)$ & $55(60)$ \\
\hline Corticosteroid therapy, $n(\%)$ & $60(66)$ \\
\hline \multicolumn{2}{|l|}{ Reason for admission } \\
\hline Coma with seizures, $n(\%)$ & $43(47)$ \\
\hline Coma without seizures, $n(\%)$ & $15(16)$ \\
\hline Acute respiratory failure, $n(\%)$ & $18(20)$ \\
\hline Shock, $n(\%)$ & $7(8)$ \\
\hline Other, $n(\%)$ & $8(9)$ \\
\hline \multicolumn{2}{|l|}{ Severity at admission } \\
\hline SAPSII & $32(18-50)$ \\
\hline SOFA & $5(3-7)$ \\
\hline \multicolumn{2}{|l|}{ Physiological variables at admission } \\
\hline Glasgow coma scale & $8(5-14)$ \\
\hline Heart rate, beats/min & $91(78-107)$ \\
\hline Systolic blood pressure, $\mathrm{mmHg}$ & $120(108-134)$ \\
\hline Respiratory rate, cycle/minute & $20(16-24)$ \\
\hline Temperature, ${ }^{\circ} \mathrm{C}$ & $37(36-37)$ \\
\hline \multicolumn{2}{|l|}{ Laboratory variables at admission } \\
\hline Leukocyte count, $10^{9} / \mathrm{L}$ & $7.8(5.5-13.2)$ \\
\hline Neutropenia, $n(\%)$ & $5(2)$ \\
\hline Serum creatinine, $\mu \mathrm{mol} / \mathrm{L}$ & $69(49-96)$ \\
\hline \multicolumn{2}{|l|}{ Arterial blood gases } \\
\hline $\mathrm{pH}$ & $7.42(7.36-7.47)$ \\
\hline $\mathrm{PaCO}_{2}, \mathrm{mmHg}$ & $37(31-42)$ \\
\hline $\mathrm{PaO}_{2} / \mathrm{FiO}_{2}$ & $305(224-387)$ \\
\hline \multicolumn{2}{|l|}{ Life-sustaining intervention } \\
\hline Mechanical ventilation, $n(\%)$ & 45 (49) \\
\hline Vasopressor, $n(\%)$ & $17(19)$ \\
\hline Renal replacement therapy, $n(\%)$ & $2(2)$ \\
\hline
\end{tabular}

Continuous variables are expressed as median (interquartile interval) and categorical variables are expressed as numbers (\%)

SAPS II Simplified Acute Physiology Score II; SOFA Sequential Organ Failure Assessment hospital lengths of stay were 3 (2-7) and 16 (8-31) days, respectively. Seventy-eight patients $(86 \%)$ were discharged alive from the ICU.

\section{Anticancer therapy after ICU discharge}

Among the 78 ICU survivors, anticancer therapy was continued in $32(41 \%)$ patients, modified in $33(42 \%)$ patients, and initiated in $13(17 \%)$ patients. Table 2 shows the factors associated with continuation or modification of anticancer therapy after ICU discharge. On multivariate logistic regression, two factors were independently associated with continuation of anticancer therapy after ICU discharge: cancer progression at ICU admission (OR 0.09, 95\% CI 0.02-0.35, $p=0.001)$ and use of corticosteroids (OR $0.07,95 \%$ CI $0.01-0.35, p=0.002$ ) at ICU admission.

\section{Mortality 1 year after ICU admission}

The mortality rate 1 year after ICU discharge was $73 \%$ (57/78 patients). Table 3 depicts the factors associated with mortality 1 year after ICU admission identified by univariate analysis. On multivariate logistic regression analysis, two factors were independently associated with lower mortality 1 year after ICU admission: continuation of anticancer therapy after ICU discharge (OR 0.18, 95\% CI 0.03-0.75, $p=0.028$ ), and Karnofsky performance status at ICU admission (OR $0.90,95 \%$ CI $0.85-0.95, p<0.001$ ).

Cumulative survival probability significantly differed between patients in whom anticancer therapy was continued, modified, or initiated (Fig. 2), with the greatest survival probability observed among patients in whom anticancer therapy was continued.

\section{Changes in performance status after ICU discharge}

Figure 3 shows changes in Karnofsky performance status from ICU admission to 1 year after ICU discharge in ICU survivors, according to management of anticancer therapy.

Karnofsky performance status was significantly different between the three anticancer therapy strategies and was the lowest in patients with anticancer therapy modified. Karnofsky performance status 1 year after ICU admission was $>60 \%$ in more than $50 \%$ of patients in whom anticancer therapy was initiated or continued.

\section{Discussion}

The main results of the study can be summarized as follows: in HGG patients discharged alive after an unplanned medical ICU stay (1), we observed a substantial proportion of survivors 1 year after ICU admission (more than one 
Table 2 Univariate analysis: factors associated with the continuation or modification of anticancer therapy in intensive care unit survivors

\begin{tabular}{|c|c|c|c|}
\hline Variables & ACT continued $(n=32)$ & ACT modified $(n=33)$ & $P$ \\
\hline Age, years & $57(50-65)$ & $57(44-66)$ & 1.000 \\
\hline Gender (male), $n(\%)$ & $20(63)$ & $22(67)$ & 0.725 \\
\hline \multicolumn{4}{|l|}{ Comorbidities } \\
\hline Chronic heart failure, $n(\%)$ & $0(0)$ & $4(12)$ & 0.114 \\
\hline Chronic respiratory disease, $n(\%)$ & $1(3)$ & $2(6)$ & 1.000 \\
\hline Charlson comorbidity index & $3(2-4)$ & $3(2-4)$ & 0.689 \\
\hline Karnofsky performance status at admission & $70(60-80)$ & $70(50-80)$ & 0.349 \\
\hline \multicolumn{4}{|l|}{ Disease status at admission } \\
\hline Cancer progression, $n(\%)$ & $13(41)$ & $26(79)$ & 0.002 \\
\hline Grade IV, $n(\%)$ & $20(63)$ & $28(85)$ & 0.040 \\
\hline IDH1/2 mutation, $n(\%)$ & $3(9)$ & $2(6)$ & 0.661 \\
\hline $1 \mathrm{p} / 19 \mathrm{q}$ codeletion, $n(\%)$ & $2(6)$ & $0(0)$ & 0.502 \\
\hline Anticancer therapy received at admission & $25(78)$ & $31(94)$ & 0.082 \\
\hline Chemotherapy, $n(\%)$ & $23(72)$ & $26(79)$ & 0.518 \\
\hline \multicolumn{4}{|l|}{ Radiotherapy, $n(\%)$} \\
\hline Corticosteroid therapy $n(\%)$ & $19(59)$ & $30(91)$ & 0.004 \\
\hline \multicolumn{4}{|l|}{ Reason for admission } \\
\hline Coma with seizures, $n(\%)$ & $18(56)$ & $14(42)$ & 0.265 \\
\hline Coma without seizures, $n(\%)$ & $3(9)$ & $5(15)$ & 0.709 \\
\hline Acute respiratory failure, $n(\%)$ & $6(19)$ & $6(18)$ & 0.953 \\
\hline Shock, $n(\%)$ & $2(6)$ & $4(12)$ & 0.672 \\
\hline Other, $n(\%)$ & $3(9)$ & $4(12)$ & 1.000 \\
\hline \multicolumn{4}{|l|}{ Severity at admission } \\
\hline SAPSII & $39(17-51)$ & $29(20-38)$ & 0.423 \\
\hline SOFA & $4(3-6)$ & $5(3-7)$ & 0.389 \\
\hline \multicolumn{4}{|l|}{ Physiological variables at admission } \\
\hline Glasgow coma scale & $9(7-14)$ & $8(6-14)$ & 0.796 \\
\hline Heart rate, beats/min & $92(80-107)$ & $97(81-105)$ & 0.948 \\
\hline Systolic blood pressure, $\mathrm{mmHg}$ & $116(105-126)$ & $130(108-141)$ & 0.056 \\
\hline Respiratory rate, cycles/minute & $20(17-22)$ & $22(19-24)$ & 0.263 \\
\hline Temperature, ${ }^{\circ} \mathrm{C}$ & $37(36-37)$ & $37(37-38)$ & 0.311 \\
\hline \multicolumn{4}{|l|}{ Laboratory variables at admission } \\
\hline Leukocyte count, $10^{9} / \mathrm{L}$ & $9.2(6.5-13,330)$ & $7.1(5.0-11.5)$ & 0.042 \\
\hline Neutropenia, $n(\%)$ & $0(0)$ & $2(6)$ & 0.492 \\
\hline Serum creatinine, $\mu \mathrm{mol} / \mathrm{L}$ & $67(50-86)$ & $70(47-100)$ & 0.778 \\
\hline \multicolumn{4}{|l|}{ Arterial blood gases } \\
\hline $\mathrm{pH}$ & $7.41(7.35-7.47)$ & $7.41(7.37-7.44)$ & 0.738 \\
\hline $\mathrm{PaCO} 2, m m H g$ & $39(33-44)$ & $36(31-41)$ & 0.299 \\
\hline $\mathrm{PaO}_{2} / \mathrm{FiO}_{2}$ & $331(274-392)$ & $300(247-380)$ & 0.487 \\
\hline \multicolumn{4}{|l|}{ Life-sustaining intervention } \\
\hline Mechanical ventilation, $n(\%)$ & $14(44)$ & $15(45)$ & 0.890 \\
\hline Vasopressor, $n(\%)$ & $3(9)$ & $8(24)$ & 0.239 \\
\hline Renal replacement therapy, $n(\%)$ & $1(3)$ & $0(0)$ & 0.492 \\
\hline
\end{tabular}

Continuous variables are expressed as median (interquartile interval) and categorical variables are expressed as numbers (\%]

SAPS II Simplified Acute Physiology Score II; SOFA Sequential Organ Failure Assessment, quarter of patients) and most of these patients exhibited relatively favorable performance status even 1 year after ICU admission, (2) continuation of anticancer therapy was possible in almost $50 \%$ of patients and was strongly associated with cancer progression and use of corticosteroids at admission, and (3) continuation of anticancer therapy and 
Table 3 Univariate analysis: factors associated with 1-year mortality in intensive care unit survivors

\begin{tabular}{|c|c|c|c|}
\hline Variables & Survivors $(n=21)$ & Non-survivors $(n=57)$ & $P$ \\
\hline Age, years & $50(39-65)$ & $56(45-67)$ & 0.207 \\
\hline Gender (male), $n(\%)$ & $12(57)$ & $36(63)$ & 0.628 \\
\hline \multicolumn{4}{|l|}{ Comorbidities } \\
\hline Chronic heart failure, $n(\%)$ & $0(0)$ & $4(7)$ & 0.569 \\
\hline Chronic respiratory disease, $n(\%)$ & $0(0]$ & $4(7]$ & 0.569 \\
\hline Charlson Comorbidity Index & $2(2-4]$ & $3(2-4]$ & 0.128 \\
\hline Karnofsky performance status at admission & $100(70-100]$ & $60(50-80]$ & $<0.001$ \\
\hline \multicolumn{4}{|l|}{ Disease status at admission } \\
\hline Cancer progression, $n(\%)$ & $6(29)$ & $33(58)$ & 0.022 \\
\hline Grade IV, $n(\%)$ & $11(52)$ & $44(77)$ & 0.033 \\
\hline IDH $1 / 2$ mutation, $n(\%)$ & $4(19)$ & $4(7)$ & 0.201 \\
\hline $1 \mathrm{p} / 19 \mathrm{q}$ codeletion, $n(\%)$ & $2(10)$ & $0(0)$ & 0.192 \\
\hline \multicolumn{4}{|l|}{ Anticancer therapy received at admission } \\
\hline Chemotherapy, $n(\%)$ & $13(87)$ & $43(75)$ & 0.239 \\
\hline Radiotherapy, $n(\%)$ & $12(80)$ & $37(65)$ & 0.529 \\
\hline Corticosteroid therapy, $n(\%)$ & $9(60)$ & $42(74)$ & 0.011 \\
\hline \multicolumn{4}{|l|}{ Reason for admission } \\
\hline Coma with seizures, $n(\%)$ & $13(62)$ & $28(49)$ & 0.316 \\
\hline Coma without seizures, $n(\%)$ & $0(0)$ & $9(16)$ & 0.103 \\
\hline Acute respiratory failure, $n(\%)$ & $3(14)$ & $11(19)$ & 0.748 \\
\hline Shock, $n(\%)$ & $3(14)$ & $3(5)$ & 0.335 \\
\hline Other, $n(\%)$ & $2(10)$ & $6(11)$ & 1.000 \\
\hline \multicolumn{4}{|l|}{ Severity at admission } \\
\hline SAPSII & $28(17-50)$ & $31(17-49)$ & 0.581 \\
\hline SOFA & $4(2-5)$ & $5(4-7)$ & 0.036 \\
\hline \multicolumn{4}{|l|}{ Physiological variables at admission } \\
\hline Glasgow coma scale & $14(7-15)$ & $8(6-13)$ & 0.029 \\
\hline Heart rate, beats/min & $89(71-101)$ & $92(80-104)$ & 0.517 \\
\hline Systolic blood pressure, $\mathrm{mmHg}$ & $116(102-122)$ & $126(108-140)$ & 0.103 \\
\hline Respiratory rate, cycles/minute & $20(16-23)$ & $20(16-24)$ & 0.883 \\
\hline Temperature, ${ }^{\circ} \mathrm{C}$ & $37(36-37)$ & $37(36-38)$ & 0.865 \\
\hline \multicolumn{4}{|l|}{ Laboratory variables at admission } \\
\hline Leukocyte count, $10^{9} / \mathrm{L}$ & $9.3(6.5-15.9)$ & $7.6(5.3-12.7)$ & 0.073 \\
\hline Neutropenia, $n(\%)$ & $1(5)$ & $1(1)$ & 0.412 \\
\hline Serum creatinine, $\mu \mathrm{mol} / \mathrm{L}$ & $65(53-84)$ & $70(47-100)$ & 0.959 \\
\hline \multicolumn{4}{|l|}{ Arterial blood gases } \\
\hline $\mathrm{pH}$ & $7.43(7.39-7.48)$ & $7.42(7.36-7.46)$ & 0.525 \\
\hline $\mathrm{PaCO}_{2}, m m H g$ & $37(35-44)$ & $37(31-41)$ & 0.622 \\
\hline $\mathrm{PaO}_{2} / \mathrm{FiO}_{2}, m m H g$ & $342(265-428)$ & $302(206-365)$ & 0.107 \\
\hline \multicolumn{4}{|l|}{ Life-sustaining intervention } \\
\hline Mechanical ventilation, $n(\%)$ & $9(43)$ & $38(54)$ & 0.368 \\
\hline Vasopressor, $n(\%)$ & $2(10)$ & $15(21)$ & 0.344 \\
\hline Renal replacement therapy, $n(\%)$ & $0(0)$ & $2(3)$ & 1.000 \\
\hline \multicolumn{4}{|l|}{ Anticancer therapy after ICU discharge } \\
\hline Continued, $n(\%)$ & $12(57)$ & $20(26)$ & 0.009 \\
\hline Modified, $n(\%)$ & $3(14)$ & $30(53)$ & 0.004 \\
\hline Initiated, $n(\%)$ & $6(29)$ & $7(12)$ & 0.091 \\
\hline
\end{tabular}

Continuous variables are expressed as median (interquartile interval) and categorical variables are expressed as numbers $(\%)$

SAPS II Simplified Acute Physiology Score II; SOFA Sequential Organ Failure Assessment. ICU intensive care unit 


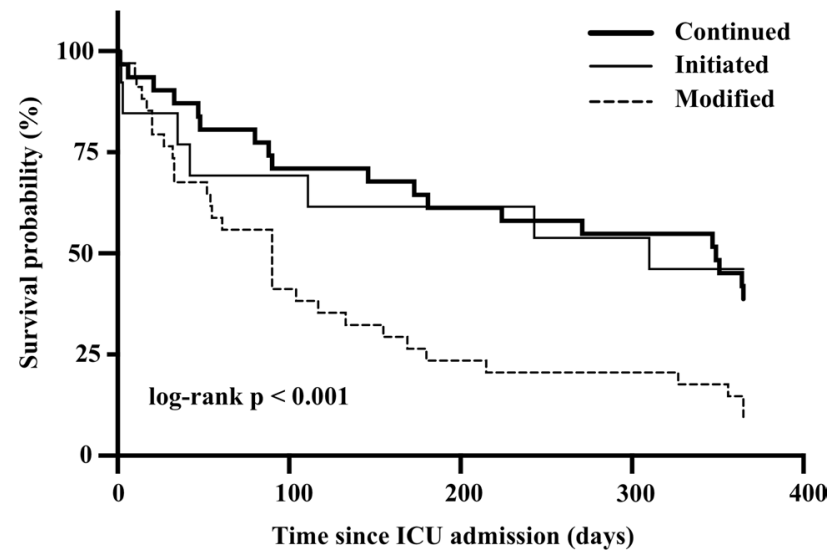

Fig. 2 One-year survival probability in high-grade glioma patients discharged alive from the intensive care unit (ICU) according to the management of anticancer therapy. Log-rank $p$ value: Initiated vs. Modified, $p=0.022$, Initiated vs. Continued, $p=0.887$, Modified vs. Continued, $p=0.001$

Karnofsky performance status at admission were associated with higher 1-year survival rates.

To the best of our knowledge, this is the first report based on a homogeneous cohort of patients with HGG discharged alive after an ICU stay, focusing on 1-year mortality, health-related functional status, and management of anticancer therapy after ICU discharge.
First of all, the low ICU mortality rate observed in this study (14\%) is consistent with recent findings, showing that ICU mortality is not higher in patients with primary malignant brain tumor than in patients with other types of solid cancer $(1,3-8)$ and patients without cancer [2, 25].

The survival rate of HGG patients 1 year after ICU admission observed in the present study was non-negligible (27\%) and most patients still presented favorable performance status at 1 year $(>60 \%)$. Indeed, considering the median time between cancer diagnosis and ICU admission [6 (2-20) months] and considering the median survival of patients with HGG [14], the $27 \%$ survival after ICU admission observed in this study appears to be substantial and encouraging. Moreover, the survival rate 1 year after ICU admission was fairly similar to that observed in patients with other types of solid cancer [2, 11, 26-29] or hematologic malignancies [30-34]. Young age, limited comorbidities, and a high proportion of rapidly reversible causes, such as seizures, could explain this relatively high 1-year survival rate. The performance status observed over the study period is consistent with a previous report of primary malignant brain tumor patients admitted to the ICU [15]. In addition, our study shows that more than one-half of patients achieved a performance status, indicating that they were able to selfcare at home (Karnofsky performance status $\geq 60 \%$ ) [22]. This is a valuable observation when assessment of functional outcome is considered to be essential to evaluate the relevance of ICU admission or maintenance of intensive therapy.
Fig. 3 Karnofsky performance status in intensive care unit (ICU) survivors at ICU admission and 1, 3, 6, 9, and 12 months after ICU admission according to the management of anticancer therapy. Anticancer therapy fixed-effect factor: $p<0.001$. Continued vs. Modified, $p=0.061$, Continued vs. Initiated, $p=0.150$, Modified vs. Initiated, $p=0.001$, Time fixed-effect factor: $p<0.001$, Patient random-effect factor: $p<0.001$
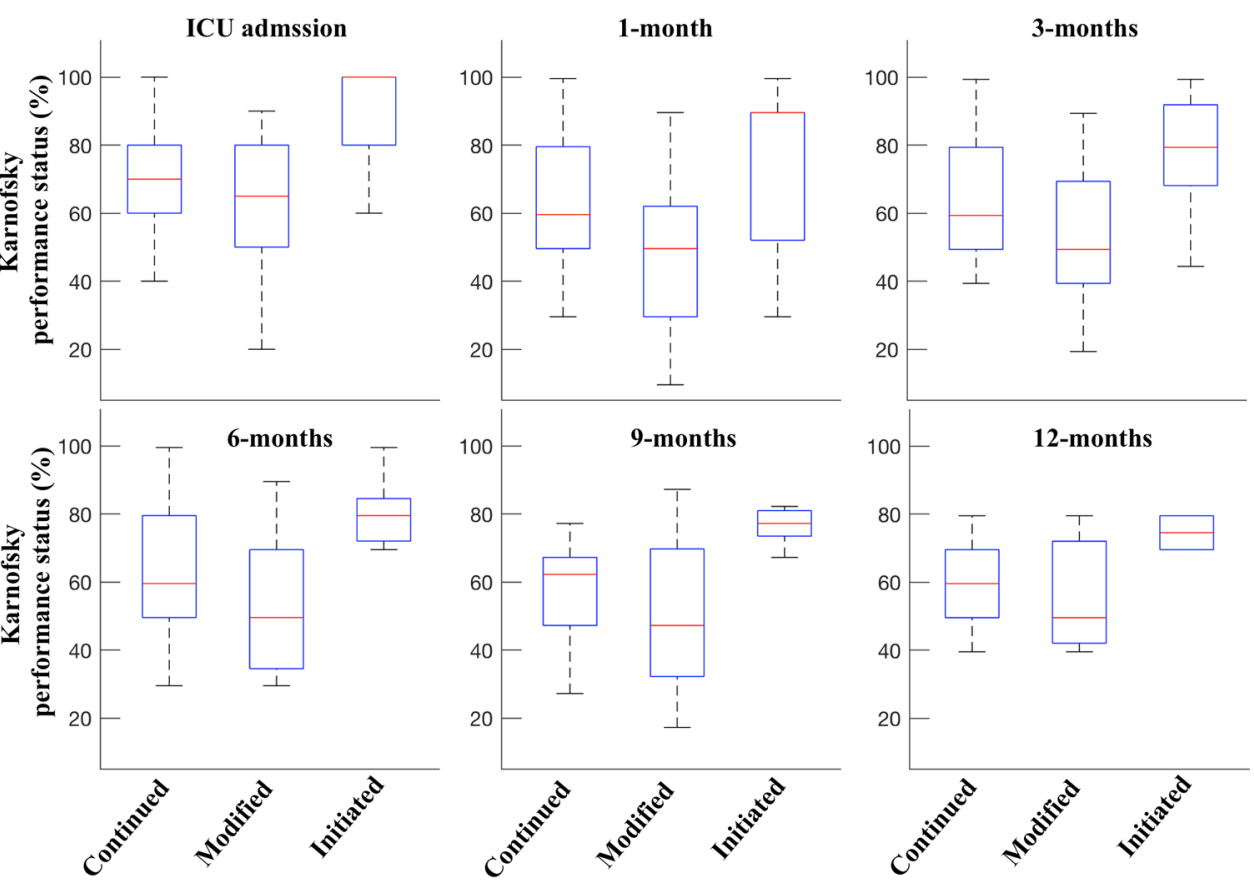

Anticancer therapy groups fixed effect: $\mathbf{p}<0.001$

-Continued vs. Modified, $\mathrm{p}=0.061$

-Continued vs. initiated, $\mathrm{p}=0.150$

-Modified vs. initiated, $\mathrm{p}=0.001$ 
To date, only a few studies have explored the impact of an ICU stay on anticancer therapy and the long-term outcome after ICU discharge [5-7, 26]. The rate of continuation of anticancer therapy observed in our study was similar to that reported in other studies [8-11]. Two factors, cancer progression and use of corticosteroids, were independently associated with failure to continue anticancer therapy in ICU survivors. While there is an obvious relationship between cancer progression and modification of anticancer therapy, the link between the use of corticosteroids and modification of anticancer therapy is less obvious and could be explained by the fact that corticosteroid prescription is generally driven by the presence of perilesional brain edema or neurological symptoms, which are both surrogates for disease activity [35]. Corticosteroid administration is a marker of poor disease control, often requiring modification of anticancer therapy. This finding is also in line with the fact that the cumulative corticosteroid dose delivered to patients with primary malignant brain tumor is associated with higher mortality [36] and decreased progression-free survival [37].

The strong influence of anticancer therapy management after ICU discharge on 1-year survival is also in line with the other reports concerning patients with solid cancer or hematologic malignancies, in whom 6-month $[9,11]$ and 1-year [10] survivals were higher in patients in whom anticancer therapy was continued after ICU discharge. Interestingly, we did not observe a higher survival rate in patients in whom anticancer therapy was initiated for a newly diagnosed cancer, which is consistent with the previous reports on patients admitted to the ICU with a newly diagnosed cancer, supporting the idea that critically ill patients with underlying undiagnosed cancer are likely to present locally advanced or metastatic disease with poor medium- [29] and long-term prognosis [27]. In these reports, Karnofsky performance status at ICU admission was also independently associated with long-term mortality [10,11].

The present study has several limitations. First, it was a retrospective study, which implies a potential bias in patient selection or data collection. However, data were extracted from a prospectively managed database and the rarity of the disease remains a major obstacle to prospective studies, even with a multicenter design. Second, the relevance of Karnofsky performance status as a health-related functional endpoint in this very specific population could be questioned. It is possible that other decisive aspects of quality of life, psychological states, and cognitive function, all likely to be impaired in HGG $[14,15]$, were ignored. Third, while we report data for patients admitted to the ICU, we did not report the proportion of HGG patients for whom ICU admission was refused during the study period, or the policies or criteria that motivated these refusals, and it is possible that patients with the poorest prognosis were, therefore, not admitted to the ICU and, thus, not included in this analysis.
Finally, because molecular testing has been systematically performed only since 2013, whereas the study period started in 2009, this study comprises many missing data and consequently failed to demonstrate any association between molecular testing and prognosis [38].

In conclusion, we report that a high proportion of $\mathrm{HGG}$ patients who survived an ICU stay may benefit from continuation of anticancer therapy after discharge, with preserved performance status, and can, therefore, expect a non-negligible survival 1 year after ICU admission. Simple factors, which can be easily identified before ICU admission, such as cancer progression, use of corticosteroids, or Karnofsky performance status at admission, are strongly associated with outcomes. If decisions concerning life-sustaining interventions are no longer considered to be futile in patients with active cancer, even metastatic cancer, a similar attitude could also be applied to HGG patients, who have probably been unreasonably denied ICU admission for many years. These results will certainly contribute to refine ICU admission policies which, in every case, should take into account the neuro-oncologists' experience and the patient's willingness.

Acknowledgements We thank Anthony Saul for his help with English style and grammar.

Author contributions MD: methodology, data curation, conceptualization, validation, writing - original draft, and writing- review and editing, NG: methodology, data curation, conceptualization, validation, and writing - original draft, software, NW: data curation, conceptualization, validation, IR: data curation, conceptualization, validation, and writing - original draft, AI: data curation, conceptualization, and validation, SD: validation, JM: data curation and validation, MD: data curation and validation, EM: data curation and validation, $\mathrm{KH}-\mathrm{X}$ : validation, TS: supervision, validation, conceptualization, and writingoriginal draft, AD: methodology, data curation, supervision, conceptualization, validation, writing — original draft, and writing-review and editing.

Funding None.

\section{Compliance with ethical standards}

Conflicts of interest Thomas Similowski reports personal fees from ADEP Assistance, AstraZeneca France, Boerhinger Ingelheim France, Chiesi France, GSK France, Lungpacer Inc., Novartis France, TEVA France, outside the submitted work; In addition, Dr. Similowski has a patent titled "brain-ventilator interface" licensed to Air Liquide Medical Systems and MyBrainTechnology, a patent for a "protection device for intubation" pending, and a patent for a "non-contact thoracic movement imaging system» pending. Alexandre Demoule reports personal fees from Medtronic, grants, personal fees, and non-financial support from Philips, personal fees from Baxter, personal fees from Hamilton, personal fees, and non-financial support from Fisher \& Paykel, grants from French Ministry of Health, personal fees from Getinge, grants and personal fees from Respinor, and grants and non-financial support from Lungpacer, outside the submitted work. Martin Dres received personal fees and travel expenses from Lungpacer outside the submitted work. Nicolas Weiss has signed research contracts with Eumedica, BMS, MedDay pharmaceuticals; he has also received personal fees 
from Norgine and Alpha-Wasserman and Consultant fees from MedDay Pharmaceuticals. Ahmed Idbaih reports research funding from $\mathrm{La}$ Fondation ARC pour la recherche sur le Cancer, Carthera, Beta-Innov, and Intselchimos; travel funding from Hoffmann-La Roche; and personal fees from Novartis, La Lettre du Cancérologue, BMS, and Cipla unrelated to the submitted work. Other authors had no conflict of interest to declare.

Ethics approval The study was approved by the French Intensive Care Society Institutional Review Board (CE SRLF 20-15) and information was given to the patients or their relatives.

Availability of data and material Our data are available to ensure transparency.

\section{References}

1. Puxty K, McLoone P, Quasim T, Sloan B, Kinsella J, Morrison DS (2015) Risk of critical illness among patients with solid cancers: a population-based observational study. JAMA Oncol 1:1078-1085

2. Vincent J-L, Marshall JC, Namendys-Silva SA, François B, Martin-Loeches I, Lipman J et al (2014) Assessment of the worldwide burden of critical illness: the intensive care over nations (ICON] audit. Lancet Respir Med 2:380-386

3. Darmon M, Bourmaud A, Georges Q, Soares M, Jeon K, Oeyen $S$ et al (2019) Changes in critically ill cancer patients' short-term outcome over the last decades: results of systematic review with meta-analysis on individual data. Intensive Care Med 45:977-987

4. Decavèle M, Rivals I, Marois C, Cantier M, Weiss N, Memasle L et al (2018) Etiology and prognosis of acute respiratory failure in patients with primary malignant brain tumors admitted to the intensive care unit. J Neurooncol 142:139-148

5. Tabouret E, Boucard C, Devillier R, Barrie M, Boussen S, Autran $D$ et al (2017) Neuro-oncological patients admitted in intensive care unit: predictive factors and functional outcome. J Neurooncol 127:111-117

6. Decavèle M, Weiss N, Rivals I, Prodanovic H, Ibdaih A, Mayaux $\mathrm{J}$ et al (2017) Prognosis of patients with primary malignant brain tumors admitted to the intensive care unit: a two-decade experience. J Neurol 264:2303-2312

7. Vincent F, Soares M, Mokart D, Lemiale V, Bruneel F, Boubaya $M$ et al (2018) In-hospital and day-120 survival of critically Ill solid cancer patients after discharge of the intensive care units: results of a retrospective multicenter study-a groupe de recherche respiratoire en réanimation en onco-hématologie (Grrr-OH) Study. Ann Intensive Care 8(1):40

8. Azoulay E, Mokart D, Pène F, Lambert J, Kouatchet A, Mayaux J et al (2013) Outcomes of critically ill patients with hematologic malignancies: prospective multicenter data from France and Belgium - a groupe de recherche respiratoire en réanimation oncohématologique study. J Clin Oncol 31:2810-2818

9. Roques S, Parrot A, Lavole A, Ancel PY, Gounant V, Djibre $\mathrm{M}$ et al (2009) Six-month prognosis of patients with lung cancer admitted to the intensive care unit. Intensive Care Med 35:2044-2050

10. Borcoman E, Dupont A, Mariotte E, Doucet L, Joseph A, Chermak A et al (2020) One-year survival in patients with solid tumours discharged alive from the intensive care unit after unplanned admission: a retrospective study. J Crit Care 57:36-41

11. Soares M, Toffart AC, Timsit JF, Burghi G, Irrazábal C, Pattison $\mathrm{N}$ et al (2014) Intensive care in patients with lung cancer: a Multinational Study. Ann Oncol 25:1829-1835
12. Shih CY, Hung MC, Lu HM, Chen L, Huang SJ, Wang JD (2013) Incidence, life expectancy and prognostic factors in cancer patients under prolonged mechanical ventilation: a nationwide analysis of 5138 cases during. Crit Care 17(4):R144

13. Hofhuis JG, Spronk PE, van Stel HF, Schrijvers GJ, Rommes JH, Bakker J (2008) The impact of critical illness on perceived health-related quality of life during ICU treatment, ICU stay, and after ICU discharge. Chest 133:377-385

14. Normilio-Silva K, de Figueiredo AC, Pedroso-de- Lima AC et al (2016) Long-term survival, quality of life, and quality-adjusted survival in critically ill patients with cancer. Crit Care Med 44:1327-1337

15. Oeyen SG, Vandijck DM, Benoit DD, Annemans L, Decruyenaere JM (2010) Quality of life after intensive care: a systemic review of the literature. Crit Care Med 38:2386-2400

16. Ehooman F, Biard L, Lemiale V, Contou D, de Prost N, Mokart $D$ et al (2019) Long-term health-related quality of life of critically ill patients with haematological malignancies: a prospective observational multicenter study. Ann Intensive Care 9(1):2

17. Taphoorn MJ, Sizoo EM, Bottomley A (2010) Review on quality of life issues in patients with primary brain tumors. Oncologist 15:618-626

18. Pill K, Jakobsen J, Christensen KB, Juhier M, Jarden M (2015) Health-related quality of life in patients with high grade gliomas: a quantitative longitudinal study. J Neurooncol 124(2):185-195

19. Omuro A, DeAngelis LM (2013) Glioblastoma and other malignant gliomas: a clinical review. JAMA 310:1842-1850

20. Louis DN, Perry A, Reifenberger G, von Deimling A, FigarellaBranger D, Cavenee WK et al (2016) The 2016 world health organization classification of tumors of the central nervous system: a summary. Acta Neuropathol 131:803-820

21. Charlson ME, Pompei P, Ales KL, MacKenzie CR (1987) A new method of classifying prognostic comorbidity in longitudinal studies: development and validation. J Chronic Dis 40:373-383

22. Le Gall JR, Lemeshow S, Saulnier FA (1993) New simplified acute physiology score (SAPS II] based on a European/North American multicenter study. JAMA 270:2957-2963

23. Vincent JL, Moreno R, Takala J, Willatts S, De Mendonça A, Bruining H et al (1996) The SOFA (Sepsis-related Organ Failure Assessment] score to describe organ dysfunction/failure. On behalf of the working group on sepsis-related problems of the European Society of intensive care medicine. Intensive Care Med 22:707-710

24. Schag CC, Heinrich RL, Ganz PA (1984) Karnofsky performance status revisited: reliability, validity, and guidelines. J Clin Oncol 2:187-193

25. Taccone FS, Artigas AA, Sprung CL, Moreno R, Sakr Y, Vincent JL (2009) Characteristics and outcomes of cancer patients in European ICUs. Crit Care 13(1):R15

26. Slatore CG, Cecere LM, Letourneau JL, O'Neil ME, Duckart JP, Wiener RS et al (2012) Intensive care unit outcomes among patients with lung cancer in the surveillance, epidemiology, and end results-medicare registry. J Clin Oncol 30:1686-1691

27. Zerbib Y, Rabbat A, Fartoukh M, Bigé N, Andréjak C, Mayaux J et al (2017) Urgent chemotherapy for life-threatening complications related to solid neoplasms. Crit Care Med 45:e640-e648

28. Vincent F, Soares M, Mokart D, Lemiale V, Bruneel F, Boubaya $M$ et al (2018) In-hospital and 120-day survival of critically ill solid cancer patients after discharge of intensive care units: results of a retrospective multicenter study-a groupe de recherche respiratoire en reanimation en onco-hématologie (Grrr-OH) study. Ann Intensive Care 8(1):40

29. Barth C, Soares M, Toffart AC, Timsit JF, Burghi G, Irrazabal C et al (2018) Lung Cancer in Critical Care (LUCCA) Study Investigators characteristics and outcome of patients with newly 
diagnosed advanced or metastatic lung cancer admitted to intensive care units (ICUs). Ann Intensive Care 8(1):80

30. Tavares M, Lemiale V, Mokart D, Pène F, Lengliné E, Kouatchet A et al (2018) Determinants of 1-year survival in critically ill acute leukemia patients: a GRRR-OH study. Leuk Lymphoma 59:1323-1331

31. Bird GT, Farquhar-Smith P, Wigmore T, Potter M, Gruber PC (2012) Outcomes and prognostic factors in patients with haematological malignancy admitted to a specialist cancer intensive care unit: a 5 yr study. Br J Anaesth 108:452-459

32. Benoit DD, Vandewoude KH, Decruyenaere JM, Hoste EA, Colardyn FA (2003) Outcome and early prognostic indicators in patients with a hematologic malignancy admitted to the intensive care unit for a life-threatening complication. Crit Care Med 31:104-112

33. Pene F, Aubron C, Azoulay E, Blot F, Thiéry G, Raynard B et al (2006) Outcome of critically ill allogeneic hematopoietic stemcell transplantation recipients: a reappraisal of indications for organ failure supports. J Clin Oncol 24:643-649
34. de Vries VA, Müller MCA, Sesmu Arbous M, Biemond BJ, Blijlevens NMA, Kusadasi N et al (2018) Time trend analysis of long-term outcome of patients with malignancies admitted at Dutch intensive care units. Br J Haematol 181:68-76

35. Kostaras X, Cusano F, Kline GA, Roa W, Easaw J (2014) Use of dexamethasone in patients with high-grade glioma: a clinical practice guideline. Curr Oncol 21:e493-503

36. Hui CY, Rudra S, Ma S, Campian JL, Huang J (2019) Impact of overall corticosteroid exposure during chemoradiotherapy on lymphopenia and survival of glioblastoma patients. J Neurooncol 143:129-136

37. Petrelli F, De Stefani A, Ghidini A, Bruschieri L, Riboldi V, Dottorini L, et al (2020) Steroids use and survival in patients with glioblastoma multiforme: a pooled analysis. J Neurol. [Online ahead of print].

38. Olar A, Wani KM, Diefes K, Heathcock LE, van Thuijl HF, Gilbert MR et al (2015) IDH mutation status and role of WHO grade and mitotic index in overall survival in grade II-III diffuse gliomas. Acta Neuropathol 129:585-596

\section{Affiliations}

\section{Maxens Decavèle ${ }^{1,2}\left(\right.$ Nicolas Gatulle $^{1} \cdot$ Nicolas Weiss $^{3,4} \cdot$ Isabelle Rivals $^{2,5} \cdot$ Ahmed Idbaih $^{6} \cdot$ Sophie Demeret $^{3}$. Julien Mayaux ${ }^{2} \cdot$ Martin Dres $^{1,2} \cdot$ Elise Morawiec $^{2} \cdot$ Khe Hoang-Xuan $^{6} \cdot$ Thomas Similowski $^{1,2} \cdot$ Alexandre Demoule $^{1,2}$}

1 Médecine Intensive Et Réanimation (Département R3S), Service de Pneumologie, AP-HP, Site Pitié-Salpêtrière, Groupe Hospitalier Universitaire APHP-Sorbonne Université, Service de Neurologie 2-Mazarin, 75013 Paris, France

2 UMRS1158 Neurophysiologie Respiratoire Expérimentale Et Clinique, INSERM, Sorbonne Université, 75005 Paris, France

3 Unité de Médecine Intensive Réanimation Neurologique, Département de Neurologie, DMU Neurosciences Et Institut de Neurosciences Translationnelles, AP-HP.Sorbonne IHU-A-ICM, Hôpital de La Pitié-Salpêtrière, Université Paris, Paris, France
4 Brain Liver Pitié-Salpêtrière (BLIPS) Study Group, INSERM UMR_S 938, Centre de Recherche Saint-Antoine, Maladies métaboliques, biliaires et fibro-inflammatoire du foie, Institute of Cardiometabolism and Nutrition (ICAN), Sorbonne Université, Paris, France

5 Equipe de Statistique Appliquée, ESPCI Paris, PSL Research University, Paris, France

6 Inserm, CNRS, UMR S 1127, Institut du Cerveau Et de La Moelle épinière, ICM, AP-HP, Hôpitaux Universitaires La Pitié Salpêtrière - Charles Foix, Service de Neurologie 2-Mazarin, Sorbonne Université, 75013 Paris, France 